\title{
Corneal Topography: A New Photokeratoscope
}

\author{
R. W. ZABEL, ${ }^{*}$ S. J. TUFT, $†$ F. W. FITZKE, J. MARSHALL \\ London
}

\begin{abstract}
Summary include

(i) the lack of topographic detail from the central cornea,

(ii) the difficulty in transporting these units,

(iii) the cost of such units.
\end{abstract}

The assessment of the topography of the cornea is an important modality in the management of surgical procedures complicated by astigmatism. Additionally, the outcome of corneal refractive surgery is often best illustrated by photokeratoscopy. Currently available photokeratoscopes suffer from a number of deficiencies that

A photokeratoscope which addresses these shortcomings is described.

Since the photokeratoscope was conceived by Placido in 1880 it has undergone a lengthy evolutionary process. The quality and dimensions of the reflected image have been improved by employing cylindrical, hemispherical, and ellipsoidal targets thereby increasing the angle subtended by the target and also partially correcting for the curvature of field of the corneal image plane..$^{2}$ In the past six years considerable advances have been made in the analysis and presentation of catoptric data. A mathematical algorithm allowing computer assisted analysis of optical power distribution has been incorporated into clinical systems by Doss and associates. ${ }^{3}$ Klyce has facilitated the visual representation of data with the development of stereo pair and distortion plot wire models that allow corneal topography to be depicted in a three dimensional form. ${ }^{4}$ Maguire and associates have developed colour-coded contour graphics that display the corneal surface as iso-power zones. $^{5}$

Although the computer generated analysis and visual display of data has advanced, presently available photokeratoscopes are large, expensive, single-function units designed primarily to demonstrate the peripheral area of the cornea for contact lens fitting applications. Desirable characteristics of a photokeratoscope would include a simple and low cost design, portability, and the ability to image both the central and peripheral cornea. With these considerations in mind we have developed a photokeratoscope that provides quality images of the corneal surface yet avoids these shortcomings.

\section{Instrumentation}

All clinical photokeratoscopes may be conceptualised as being constructed of several individual components. These include the

\footnotetext{
* Supported by the A. E. Baker Foundation.

$\dagger$ Supported by the T.F.C. Frost Charitable Trust.

Presented at the Ophthalmological Society of the United Kingdom meeting, April 1988, Harrogate, UK. From Departments of Clinical Ophthalmology and Visual Science.

Correspondence to R. Zabel, Institute of Ophthalmology, Judd Street, London, UK WC1H 9QS.
} 


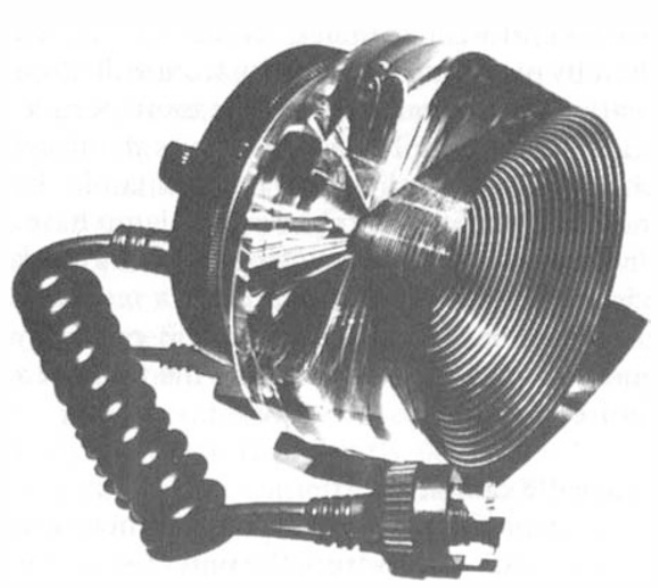

Fig. 1. The photokeratoscope body was lathed from a block of perspex. A ring flash with built in modelling lights is shown attached to the back surface. This assembly is in turn threaded onto a fixed-focus $80 \mathrm{~mm}$ macro lens. A split prism view finder facilitates focusing by eliminating operator accommodation.

photokeratoscope body which consists of the faceplate of concentric rings that are imaged by the cornea, a system to illuminate the rings of the photokeratoscope and the cornea, a means to document the corneal topography, and often a method for the analysis of the catoptric image. In order to incorporate simplicity and portability into the design of our photokeratoscope, readily available photographic equipment was used for the illuminating and recording systems (Olympus T10 ring flash and Olympus OM2/spot camera body). The dimensions of the photokeratoscope body were therefore dependent upon the dimensions of the ring flash and upon the pattern of light emitted by the flash element. The photokeratoscope body that evolved from these design restrictions is shown in Fig. 1 (with the ring flash attached to the back surface). The device was precision lathed from a block of solid perspex and comprises a faceplate of 29 rings, $0.4 \mathrm{~mm}$ in diameter spaced on $2.0 \mathrm{~mm}$ centres, and angulated medial and lateral surfaces. Figure 2 shows a schematic of the ring flash and the photokeratoscope body with three illustrative light rays. The angulation of the medial and lateral walls was chosen such that the angle at which light rays 1 and 3 are incident on these surfaces is greater than the threshold for total internal reflection. In this way, light produced by the ring flash that would otherwise not have illuminated the cornea is efficiently redirected by the angulated surfaces of the photokeratoscope body.

Based on the assumption of spherical corneal symmetry, Ludlam and Wittenberg have shown that the shape of the target locus required to produce a planar image upon reflection from the corneal tear film has a near elliptical form. ${ }^{2}$ The target surface used in the design of the initial prototype was a truncated cone that approximated this ellipse. In corneas that significantly deviate from sphericity however a large discrepency arises between the configuration of the abnormal cornea relative to the photokeratoscope faceplate that is required to produce a flat image plane upon reflection. In advanced keratoconus for example, the result is that either the central or the peripheral rings, but not both simultaneously, may be focused. ${ }^{6}$ To extend the range of the photokeratoscope and allow the imaging of these grossly non-spherical corneal surfaces the apical angulation of the cone was decreased further better to conform to these conical corneas. As shown in Fig. 2, the face-
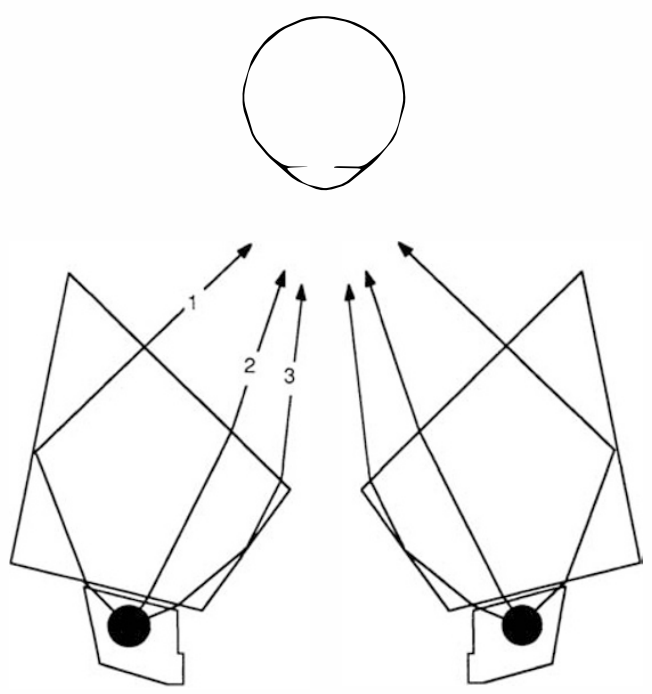

Fig. 2. Light leaves the ring flash to reach the cornea either directly (ray 2) or after total internal reflection and refraction (rays 1 and 3). The image of the rings formed in the corneal image plane just anterior to the plane of the iris is then visualised through the apical aperture and photographed. 


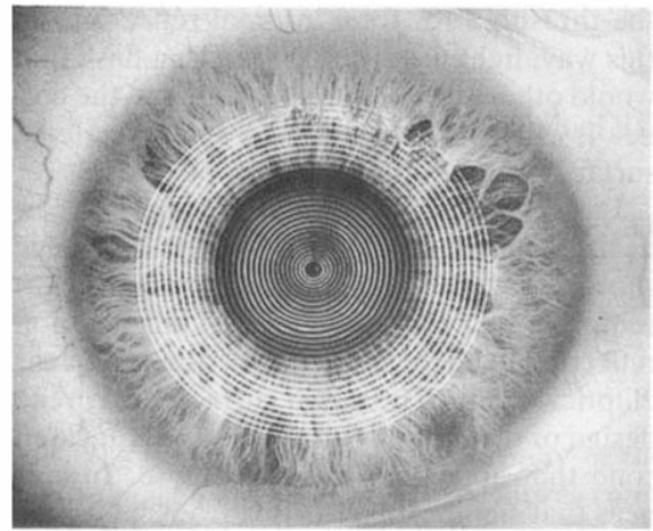

Fig. 3. Photokeratoscope picture of a normal human cornea (see text for details).

plate forms a cone with an apical angle of $90^{\circ}$. In the keratoconus patients thus far studied all of the rings have been in focus simultaneously.

The radius of the base of the faceplate cone was chosen so that when in use at a comfortable working distance the images of the outer rings lie in the mid-periphery of the cornea. On a cornea of average surface power the outermost ring has a diameter of $\sim 8.5 \mathrm{~mm}$.

The optical zone of the cornea is located centrally within the cornea and has a diameter of 3-4 $\mathrm{mm}$. The diameter of the central ring of photokeratoscope pictures is proportional to the diameter of the apical aperture of the photokeratoscope body. In order to maximise the coverage of this area and thereby provide maximal clinical information from the optical zone of the cornea the diameter of the apical aperture of the photokeratoscope should be minimised. A reduction in aperature size does however interfere with the light requirements of the camera. The optimum diameter of the central aperture was found to be $6.0 \mathrm{~mm}$, a compromise that results in the innermost ring on photokeratoscope pictures having a diameter of $\sim 0.3 \mathrm{~mm}$.

A fixed focus $80 \mathrm{~mm}$ macro lens and an auto bellows attach the photokeratoscope body to the camera which has an automatic light metering feature. The magnification and working distance of the photokeratoscope could be regulated by adjusting the extension of the camera bellows. In this manner the area of the cornea utilised in the formation of the photokeratoscope image could be varied thereby providing information from either the central optical zone or from the more peripheral limbal areas. The assembly was stabilised on a focusing rail which in turn could be mounted on either a tripod or slit-lamp base. Photographs were taken using 400 ASA black and white film. The software for a real-time computer based analysis system is presently under development and will be the topic of a future report.

\section{Clinical Examples}

1. Normal Cornea (Fig. 3) On a cornea with average radii of curvature the outer ring spans $\sim 8.5 \mathrm{~mm}$ while the inner ring has a diameter of $\sim 0.3 \mathrm{~mm}$. The ring aberrations seen below the visual axis are not consistently present and we feel therefore that they represent local differences in tear film thickness.

2. Penetrating Keratoplasty (Fig. 4) The central rings within the image are nearly circular whereas towards the periphery a definite angulation is noted. While correction of the full manifest refraction of $-3.00+4.00 \times 120$ gave a visual acuity of $6 / 9$, the photokeratoscope picture suggests that this degree of astigmatic correction is not required. A visual acuity of $6 / 9$ was in fact obtained with a -3.00 diopter sphere.

\section{Discussion}

We have demonstrated that this photokeratoscope can produce high quality images from

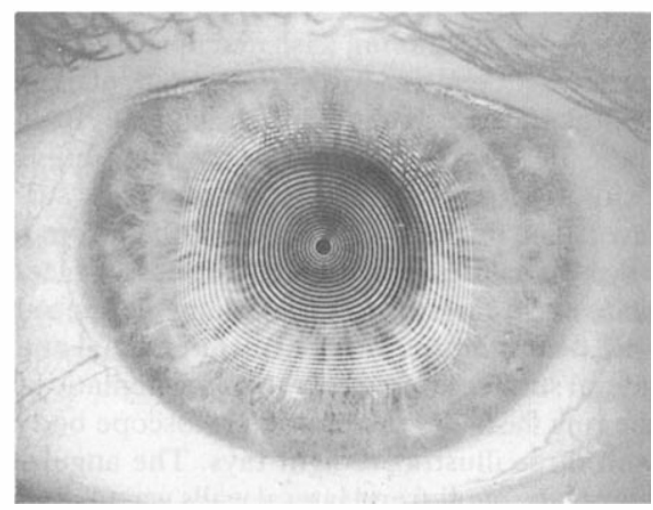

Fig. 4. Photokeratoscope picture of a patient following a penetrating keratoplasty (see text for details). 
the surface of both normal and abnormal human corneas. In addition to the clinical examples contained in this report we have also used the photokeratoscope to obtain topographic information from patients with keratoconus, idiopathic astigmatism, and epikeratophakia lenticules. Our animal studies on excimer laser photorefractive keratectomy have benefitted by the detailed topographic information from the central cornea provided by photokeratoscopy. A computer assisted analysis system is presently being developed to quantify the catoptric imagery. However even without complex computer graphics the expertise required to interpret most clinical problems is quickly gained. We anticipate this photokeratoscope will provide access to low cost corneal topographic analysis for the management and investigation of astigmatism and refractive surgery.
We are indebted to Mr. Paul Johnson for providing technical expertise.

\section{References}

${ }^{1}$ Marshall J, Trokel S, Rothery S, Krueger R: Photoablative reprofiling of the cornea using an excimer laser: Photorefractive keratectomy. Lasers Ophthalmol 1986, 21: 1-??.

${ }^{2}$ Ludlum WM and Wittenberg S: Measurements of the ocular dioptric elements utilizing photographic methods. Part II. Cornea-theoretical considerations. Am J Optom 1966, 42: 249-000.

${ }^{3}$ Doss JD, Hutson RL, Rowsey JJ, Brown R: Method for calculation of corneal profile and power distribution. Arch Ophthalmol 1981, 99: 1216-0000.

${ }^{4}$ Klyce SD: Computer-assisted cornela topography. Invest Ophthalmol Vis Sci 1984, 25: 1426-0000.

${ }^{5}$ Maguire LJ, Singer DE, Klyce SD: Graphic presentation of computer-analysed keratoscope photographs. Arch Ophthalmol 1987, 105: 223-000.

${ }^{6}$ Dietze TR and Durrie DS: Indications and treatment of keratoconus using epikeratophakia. Ophthalmol 1988, 95: 236-000. 